\title{
Design of an Autonomous DNA Nanomechanical Device Capable of Universal Computation and Universal Translational Motion ${ }^{\star}$
}

\author{
Peng Yin ${ }^{1}$, Andrew J. Turberfield ${ }^{2}$, Sudheer Sahu ${ }^{1}$, and John H. Reif ${ }^{1}$ \\ 1 Department of Computer Science, Duke University \\ Box 90129, Durham, NC 27708-0129, USA. \\ \{py, sudheer, reif\}@cs.duke.edu \\ 2 University of Oxford, Department of Physics, Clarendon Laboratory \\ Parks Road, Oxford OX 1 3PU, UK. \\ a.turberfieldaphysics.ox.ac.uk
}

\begin{abstract}
Intelligent nanomechanical devices that operate in an autonomous fashion are of great theoretical and practical interest. Recent successes in building large scale DNA nano-structures, in constructing DNA mechanical devices, and in DNA computing provide a solid foundation for the next step forward: designing autonomous DNA mechanical devices capable of arbitrarily complex behavior. One prototype system towards this goal can be an autonomous DNA mechanical device capable of universal computation, by mimicking the operation of a universal Turing machine. Building on our prior theoretical design and prototype experimental construction of an autonomous unidirectional DNA walking device moving along a linear track, we present here the design of a nanomechanical DNA device that autonomously mimics the operation of a 2-state 5-color universal Turing machine. Our autonomous nanomechanical device, called an Autonomous DNA Turing Machine (ADTM), is thus capable of universal computation and hence complex translational motion, which we define as universal translational motion.
\end{abstract}

\section{Introduction}

\subsection{Previous and Current Work}

DNA has been explored as an excellent material for building large scale nano-structures, constructing individual nanomechanical devices, and performing computations [26]. Recent years have seen substantial progress in these three fields and this progress provides a solid foundation for the next step forward: designing (and implementing) autonomous nanomechanical devices capable of arbitrarily complex behavior, e.g. motion and computation. A major challenge in nanoscience is to design a nanomechanical device that is capable of universal translational motion, which we define as the motion determined by the head of a universal Turing machine. To meet this challenge, we describe the construction of a nanomechanical device embedded in a DNA lattice that

\footnotetext{
${ }^{\star}$ Extended abstract. For full version, see [41].
} 
mimics the operation of a universal Turing machine in an autonomous fashion. We call this prototype device an Autonomous DNA Turing Machine, or ADTM. The design of ADTM benefits from recent progress in the three aforementioned fields, can be viewed as an exciting synergistic point of the three fields, and in turn contributes to the advance of each of these fields: it can be viewed as an autonomous intelligent nano-lattice, an autonomous intelligent nanorobotics device, and a compact autonomous universal computing device.

Large scale periodic lattices have been made from a diverse set of branched DNA molecules, such as the DX molecules [35], TX molecules [10], rhombus molecules [17], and $4 \mathrm{x} 4$ molecules [38]. In addition, researchers have also successfully constructed aperiodic programmable DNA lattices [37]. These self-assembled lattices provide a structural base for our construction of ADTM, whose main structure can be implemented as two parallel arrays of DNA molecules embedded in a one-dimensional DNA lattice.

A variety of DNA nanomechanical devices have been previously constructed that demonstrate motions such as open/close $[14,28,29,43]$, extension/contraction $[2,8$, $12]$, and rotation [18,39], mediated by external environmental changes such as the addition and removal of DNA fuel strands $[2,8,12,28,29,39,43]$ or the change of ionic composition of the solution $[14,18]$. A desirable improvement of these devices is the construction of DNA nanomechanical devices that achieve motions beyond the non-autonomous and localized non-extensible motions exhibited by the above devices. There have already been some exciting work in this direction. Turberfield and colleagues have designed a free running DNA machine [31] which uses DNA as fuels and demonstrates autonomous motion. Mao's group recently demonstrated an autonomous DNA motor powered by a DNA enzyme [6]. Seeman's group has constructed a DNA walking device controlled by DNA fuel strands [27]. Reif has designed an autonomous DNA walking device and an autonomous DNA rolling device that move in a random bidirectional fashion along DNA tracks [23]. The authors have recently designed autonomous DNA walking devices capable of autonomous programmable unidirectional motions along linear tracks $[40,42]$. One of these prototype unidirectional DNA walking devices has also been experimentally constructed in our lab [42]. However, these devices only exhibit simple unidirectional motion.

A rich family of DNA computation schemes have been proposed and implemented [7, $11,13,15,16,19, ?, 22,20,24,25,30,34]$ following Adleman's seminal report in 1994 [1]. Among them, the most relevant work that has inspired the construction here is the universal DNA Turing machine design by Rothemund [24] and the autonomous 2-state 2-color finite state automata constructed by Shapiro's group [3-5]. In Rothemund's innovative design, the transition table of a universal Turing machine is encoded in a circular DNA and the encoded transitions are carried out by enzymic cleavages and ligations. However, these reactions need to be carried out manually for each transition. In contrast, the ADTM described here operates in an autonomous fashion with no external environmental mediation. In the inspiring construction by Shapiro's group, a duplex DNA encoding the sequence of input symbols is digested sequentially by an endonuclease in a fashion mimicking the processing of input data by a finite state automaton. Some of their encoding schemes are used in the construction described in this paper, i.e. using DNA sticky end to encode the combination of state and symbol and using a class 
II restriction enzyme to effect state change. A limitation of the finite state automata construction is that the data are destroyed as the finite state automaton proceeds. Though this feature does not affect the proper operation of a finite state automaton, it poses a barrier to further extending the finite state automaton to more powerful computing devices such as Turing machines.

In this work, we encode computational power into a DNA walking device embedded in a DNA lattice and thus accomplish the design for an autonomous nanomechanical device capable of universal computation, by mimicking the operation of a 2-state 5-color universal Turing machine. In the process of computation, the device can also demonstrate universal translational motion as defined above, i.e. the motion demonstrated by the head of universal Turing machine.

\subsection{Universal Turing Machine}

A Turing machine is a theoretical computational device invented by Turing for performing mechanical or algorithmic mathematical calculations $[32,33]$. Though the construction and operational rules of a Turing machine may seem beguilingly simple and rudimentary, it has been shown that any computational process that can be done by present computers can be carried out by a Turing machine.

A Turing machine consists of two parts, a read-write head and a linear tape of cells encoding the input data. The head has an internal state $q$ and each cell has a color (or data) $c$ (as described in [36]). At any step, the head resides on top of one cell, and the color of that cell and the state of the head together determines a transition: (i) the current cell may change to another color; (ii) the head may take a new state; (iii) the head may move to the cell immediately to the left or the right of the current cell.

A universal Turing machine is a Turing machine that can simulate the operation of any other Turing machine. Let $m$ and $n$ be the number of possible states and the number of possible colors of a Turing machine, respectively. The Turing machine with a proven universal computation capacity and the smallest $m \times n$ value is a 2-state 5color Turing machine described in [36]. In this paper, we describe the design of a DNA nanomechanical device that simulates the general operation of an arbitrary 2-state 5color Turing machine whose head moves to either its left or right neighbor in every transition, and in particular, the universal Turing machine described in [36].

The rest of the paper is organized as follows. We give a structural overview of ADTM in Sect. 2 and an operational overview in Sect. 3, followed by a detailed stepby-step molecular implementation of the operation in Sect. 4. In Sect. 5, we briefly overview two major technical challenges in designing ADTM. We close in Sect. 6 with a discussion of our results.

\section{Structural Overview}

Figure 1 illustrates the structure of ADTM. ADTM operates in a solution system. The major components of ADTM are two parallel arrays of dangling molecules tethered to two rigid tracks. The two rigid tracks can be implemented as rigid DNA lattices as described in Sect. 1.1, for example, the rhombus lattice [17] as shown in Figure 1. A 


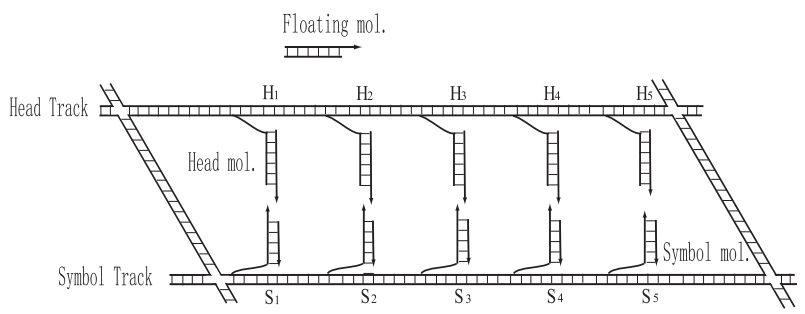

Fig. 1. Schematic drawing of the structure of ADTM. $H_{i}$ and $S_{i}$ denote Head-Molecule and Symbol-Molecule, respectively. The backbones of DNA strands are depicted as line segments. The short bars represent base pairing between DNA strands

dangling-molecule is a duplex DNA fragment with one end linked to the track via a flexible single strand DNA and the other end possessing a single strand DNA extension (the sticky end). Due to the flexibility of the single strand DNA linkage, a dangling-molecule moves rather freely around its joint at the track. The upper and lower arrays of danglingmolecules are called Head-Molecules, denoted as H, and Symbol-Molecules, denoted as $S$, respectively. We require that the only possible interactions between two danglingmolecules are either a reaction between a Head-Molecule and the Symbol-Molecule immediately below it or a reaction between two neighboring dangling-molecules along the same track. This requirement can be ensured by the rigidity of the tracks and the properly spacing of dangling-molecules along the rigid tracks.

In addition to the two arrays of dangling-molecules, there are floating-molecules. A floating-molecule is a free floating (unattached to the tracks) duplex DNA segment with a single strand overhang at one end (sticky end). A floating-molecule floats freely in the solution and thus can interact with another floating-molecule or a dangling-molecule provided that they possess complementary sticky ends. There are two kinds of floatingmolecules: the Rule-Molecules and the Assisting-Molecules. The Rule-Molecules specify the computational rules and are the programmable part of ADTM while the AssistingMolecules assist in the carrying out the operations of ADTM, as described in detail later.

The array of Symbol-Molecules represent the data tape of a Turing Machine; the array of Head-Molecules represent the moving head of a Turing Machine (more specifically, at any time, only one Head-Molecule is active, and its position indicates the position of the head of a Turing Machine); the Rule-Molecules collectively specify the transition rules for ADTM; the Assisting-Molecules are auxiliary molecules that assist in maintaining the operation of ADTM.

The duplex portion and/or the sticky end of a DNA molecule may encode the following information: (i) state, the Turing machine state; (ii) color, the color (data) encoded in a symbol molecule; (iii) position, the position type of a Head-Molecule. The state, color, and position information are denoted as $q, c$, and $p$, respectively, where $q \in$ $\left\{Q_{A}=L O N G, Q_{B}=S H O R T\right\}, c \in\left\{C_{A}, C_{B}, C_{C}, C_{D}, C_{E}\right\}, p \in\left\{P_{A}, P_{B}, P_{C}\right\}$ for the 2-state 5-color ADTM. The position information $p$ indicates the position type of a Head-Molecule. This information is essential for dictating the bidirectional motion 
of the head. An information encoding molecule is denoted as $X^{a}[y]^{b}$, where $X$ is its duplex portion, $[y]$ is its sticky end portion; $a$ is the state/color/position information encoded in $X$, and $b$ is the state/color/position information encoded in $[y]$. A complementary sticky end of $[y]$ is denoted as $[\bar{y}]$.

The array of Head-Molecules is denoted as $\left(H_{1}, H_{2}, H_{3}, \ldots\right)$; the array of SymbolMolecules is denoted as $\left(S_{1}, S_{2}, S_{3}, \ldots\right)$. To specify the motion of ADTM head, we have Head-Molecules arranged in periodic linear order along the Head-track

$$
\left(H_{1}^{P_{A}}, H_{2}^{P_{B}}, H_{3}^{P_{C}}, H_{4}^{P_{A}}, H_{5}^{P_{B}}, H_{6}^{P_{C}} \cdots\right)
$$

\section{Operational Overview}

At the beginning of a transition operation of ADTM, all the Symbol-Molecules possess sticky ends $[\bar{s}]$. A Symbol-Molecule with a sticky end $[\bar{s}]$ is referred to as in its default configuration; the $[\bar{s}]$ sticky end is referred to as a default sticky end. One of the HeadMolecules encodes the current state of ADTM in its duplex portion and possesses an active sticky end $[s]$ that is complementary to the sticky end $[\bar{s}]$ of the Symbol-Molecule just below it. This Head-Molecule is referred to as the active Head-Molecule. In contrast, all other Head-Molecules (with sticky ends other than $[s]$ ) are in default or inactive configuration.

Figure 2 gives a high level description of the events that occur during one transition of ADTM. For ease of exposition, we describe the operation in 4 stages. The 8 types of ligation events that correspond to the detailed 8-step implementation of ADTM (Sect. 4) are also marked in the figure to assist the reader in relating the high level description in this section to detailed step-by-step implementation in Sect. 4. In Stage 1, the active Head-Molecule (labeled with a triangle, $H_{3}$ in the example shown in Figure 2) is ligated to the Symbol-Molecule ( $S_{3}$ in Figure 2 ) directly below it, creating an endonuclease recognition site in the ligation product (event (1) in Figure 2). The ligation product is subsequently cleaved into two molecules by an endonuclease. The sticky end of each of the two newly generated molecules encodes the current state and the current color of ADTM.

In Stage 2, both the new Symbol-Molecule and the new Head-Molecule are ligated to floating Rule-Molecules (events (2) and (4) in Figure 2), which possess complementary sticky ends to them and correspond to one entry in the Turing machine transition table. The ligation product between the Symbol-Molecule and the Rule-Molecule is in turn cleaved, generating a new Symbol-Molecule dictated by the current state and color information as well as the transition rule. The new Symbol-Molecule encodes the new color in its sticky end. Similarly, the ligation product between the Head-Molecule and the Rule-Molecule is cleaved, generating a new Head-Molecule whose duplex portion encodes information of Turing machine's next state and whose sticky end encodes the moving direction of the head.

In Stage 3, the newly generated Symbol-Molecule is further modified by an AssistingMolecule so that it will encode the new color in its duplex portion (rather than sticky end) and possess an $[\bar{s}]$ sticky end (event (3) in Figure 2). The sticky end of the HeadMolecule will dictate it to hybridize with either the Head-Molecule to its left or to its 


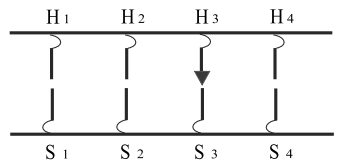

Stage 1
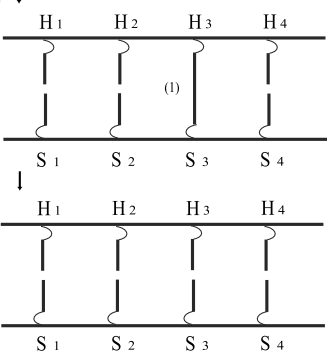

Stage 2

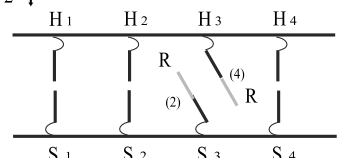

1

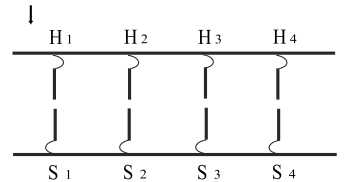

Stage 3
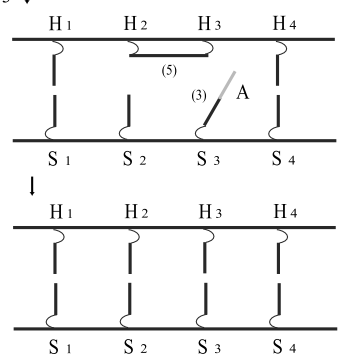

Stage 4

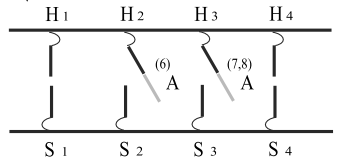

西

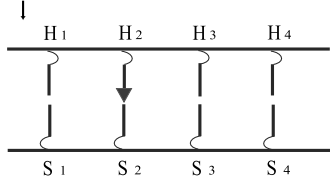

Fig. 2. Operational overview of ADTM. The dangling Head-Molecules and Symbol-Molecules are depicted as dark line fragments. The floating Rule-Molecules and Assisting-Molecules are depicted as light colored light segments. $H, S, R$, and $A$ denote Head-Molecule, Symbol-Molecule, Rule-Molecule, and Assisting-Molecule, respectively. The triangle indicates the active HeadMolecule. $(i)$ indicates the ligation event that occurs in Step $i$ as will be described in the detailed step-by-step implementation of ADTM (Sect. 4)

right, depending on which of its neighbors possesses a complementary sticky end (event (5) in Figure $2, H_{3}$ is ligated with its left neighbor $H_{2}$ ). Next, the ligation product between these two Head-Molecules is cleaved.

In Stage 4, the two Head-Molecules are modified by floating Assisting-Molecules (events $(6)$ and $(7,8)$ in Figure 2) so that the first Head-Molecule is restored to its inactive configuration (with a default sticky end) and the second Head-Molecule encodes the state information in its duplex part and possesses an active sticky end $[s]$ and thus becomes an active Head-Molecule, ready to interact with the Symbol-Molecule located directly below it.

This finishes a transition and the operation can thus go on inductively. We emphasize that we describe the events in stages only for ease of exposition. The proper operation of ADTM does not require the synchronization of the events as described above. For example, event (3) in Stage 3 can occur either before event (4) or after event (8). 


\section{Step-by-step Implementation}

We next give a detailed 8-step description of the operation of ADTM. Each step consists of ligation and cleavage events. The ligation events are marked in Figure 2 with $(i)$, where $i=1,2, \ldots, 8$. To demonstrate the practicality of our design, we give full DNA sequence for the reactions of each step. In addition to the $A, T, C$, and $G$ bases, we also occasionally require another pair of unnatural bases which we denote as $E$ and $F$. The reason to use $E$ and $F$ is to minimize the futile reactions as described later and hence increase the efficiency of our ADTM. The practicality of use of $E$ and $F$ is justified by the existing technology to make such bases and incorporate them into DNA strands. For a recent survey on unnatural bases, see [9].

At the start of the operation of ADTM, the configuration of the Head-Molecules array along the Head-track is

$$
\left(\hat{H}_{1}^{p_{1} q}[s]\right)\left([\bar{h}]^{p_{2}} H_{2}^{p_{2}}\right)\left([\bar{h}]^{p_{3}} H_{3}^{p_{3}}\right) \ldots
$$

where $p_{i}=P_{A}$ for $i=3 k+1, p_{i}=P_{B}$ for $i=3 k+2, p_{i}=P_{C}$ for $i=3 k+3$ for $k=0,1,2, \ldots$ The first Head-Molecule is special: it is the active Head-Molecule and represents the current position of the active head. We use the symbol ${ }^{\wedge}$ to denote the active configuration of a Head-Molecule. $H_{1}$ has the unique sticky end $[s]$, which is complementary to the sticky end $[\bar{s}]$ of a Symbol-Molecule in default configuration (in particular, the Symbol-Molecule directly below it). Thus, $H_{1}$ can hybridize and be ligated with Symbol-Molecule $S_{1}$, and this will start the operation of the Turing machine. Recall that $p$ encodes the position type information of a Head-Molecule. This position type information is encoded both in the sticky end portion and in the duplex portion of a Head-Molecule. As we will see below, the sticky end encoding of $p$ is necessary for dictating the appropriate motion of an active head; the duplex portion encoding is necessary for restoring a Head-Molecule to its default configuration after it turns from an active to an inactive state.

The Symbol-Molecules array along the Symbol-track is

$$
\left([\bar{s}] S_{1}^{c_{1}}\right)\left([\bar{s}] S_{2}^{c_{2}}\right)\left([\bar{s}] S_{3}^{c_{3}}\right) \ldots
$$

All the Symbol-Molecules have the same sticky end $[\bar{s}]$. As such, whenever a HeadMolecule directly above a Symbol-Molecule becomes active, this Symbol-Molecule can interact with the active Head-Molecule. Note that $[\bar{s}]$ encodes no color information - the color information $c_{i}$ is instead encoded completely in the duplex portion of a Symbol-Molecule.

\subsection{Reaction between a Head-Molecule and a Symbol-Molecule}

Step 1. In step 1, the active state-encoding Head-Molecule is first ligated with the colorencoding Symbol-Molecule below it, and then the ligation product is cut into a new Head-Molecule and a new Symbol-Molecule, the sticky ends of which both encode the current state and color information.

Let $\hat{H}_{i}^{p q}[s]$ be the current active head (encoding position type $p$ and current state $q$ ); let $[\bar{s}] S_{i}^{c}$ be the Symbol-Molecule below it (encoding current color $c$ ). $\hat{H}_{i}$ and $S_{i}$ 


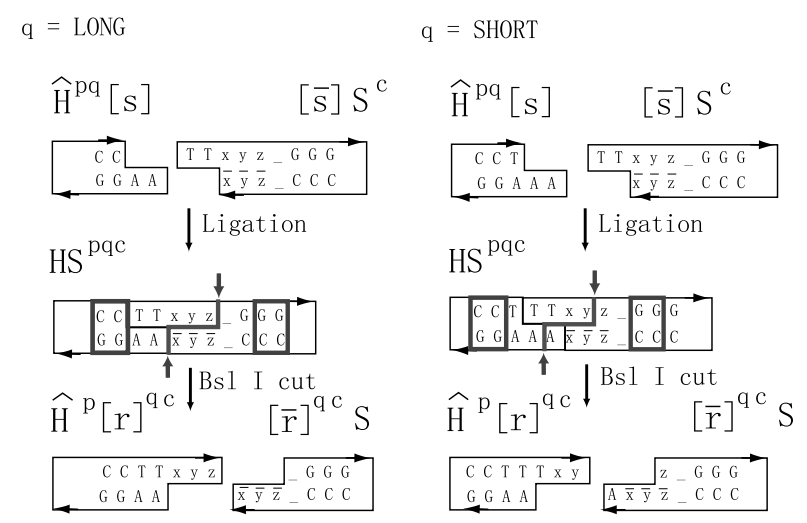

Fig. 3. Step 1 of the operation of ADTM. The current state $q$ and color $c$ are initially encoded in the duplex portion of the Head-Molecule and the Symbol-Molecule, respectively. After the ligation and cleavage, both the sticky ends of the new Head-Molecule and Symbol-Molecule encode the current state $q$ and the current color $c$. The encoding scheme of $c$ is described in Table 1. Bsl I recognition sites and cleavage sites are indicated with boxes and pairs of bold arrows, respectively

has complementary sticky ends and hence these two are ligated into $\left(H_{i} S_{i}\right)^{p q c}$. An endonuclease recognizes the newly formed recognition site in the ligation product and cuts the ligation product into $\hat{H}_{i}^{p}[r]^{q c}$ and $[\bar{r}]^{q c} S_{i}$. Now the sticky ends of both $\hat{H}_{i}$ and $S_{i}$ encode the current color and state. Step 1 can be described by the following equation,

$$
\hat{H}_{i}^{p q}[s]+[\bar{s}] S_{i}^{c} \rightarrow\left(H_{i} S_{i}\right)^{p q c} \rightarrow \hat{H}_{i}^{p}[r]^{q c}+[\bar{r}]^{q c} S_{i}
$$

The first part of the equation is the ligation of Head-Molecule $\hat{H}_{i}{ }^{p q}[s]$ with SymbolMolecule $[\bar{s}] S_{i}^{c}$ into $\left(H_{i} S_{i}\right)^{p q c}$; the second part is the cleavage of the ligation product into Head-Molecule $\hat{H}_{i}{ }^{p}[r]^{q c}$ and Symbol-Molecule $[\bar{r}]^{q c} S_{i}$. Note that now both the sticky ends of the Head-Molecule and the Symbol-Molecule are encoding the current state and color. This encoding scheme is in the same spirit as the one used in [5].

Figure 3 gives the molecular implementation of Step 1. For simplicity, only the relevant end sequences are given. The encoded information $p$ is not shown. Both the case when $q=S H O R T$ and the case when $q=L O N G$ are depicted. $x y z$ is the color encoding region for Symbol-Molecule $S$. The encoding scheme used is shown in Table 1.

\subsection{Color Change of a Symbol-Molecule}

After Step 1, the sticky end of $[\bar{r}]^{q c} S_{i}$ encodes the current state and color. This sticky end is subsequently detected by a Rule-Molecule $\tilde{R}[r]^{q c}$, which has a complementary sticky end. $\tilde{R}[r]^{q c}$ corresponds to one entry in the transition table for ADTM, and determines the next color $c^{\prime}$ that will be encoded in $S_{i}$. This color transition occurs in Step 2 and $S_{i}$ is modified to possess a sticky end $[\bar{e}]^{c^{\prime}}$ that encodes the new color $c^{\prime}$. In Step 
Table 1. The molecular implementation of the color encoding scheme of a Symbol-Molecule. $c$ is the color; $x y z$ is the sticky $[r]$ exposed when state $q=L O N G$; T $x y$ is the sticky end $[r]$ when state $q=S H O R T$. Note that all the ten sticky end sequences are different from each other

\begin{tabular}{|c|c|c|c|c|c|c|}
\hline & $c$ & $C_{A}$ & $C_{B}$ & $C_{C}$ & $C_{D}$ & $C_{E}$ \\
\hline$q=L O N G$ & $x y z$ & TTA & CTT & CAA & AEA & CEA \\
\hline$q=S H O R T$ & T $x y$ & TTT & TCT & TCA & TAE & TCE \\
\hline
\end{tabular}

Table 2. The relation between the length of the spacer, the sequence of sticky end $[\bar{e}]$ and the new color of a Symbol-Molecule. $l$ is the spacer length; $\bar{e}$ is the sticky end sequence; $c^{\prime}$ is the new color

\begin{tabular}{|c|c|c|c|c|c|c|}
\hline & $c^{\prime}$ & $C_{A}$ & $C_{B}$ & $C_{C}$ & $C_{D}$ & $C_{E}$ \\
\hline & $\bar{e}$ & $\mathrm{CA}$ & $\mathrm{AC}$ & $\mathrm{CT}$ & $\mathrm{TT}$ & $\mathrm{TG}$ \\
\hline$q=$ LONG & $l$ & 8 & 7 & 6 & 5 & 4 \\
\hline$q=$ SHORT & $l$ & 7 & 6 & 5 & 4 & 3 \\
\hline
\end{tabular}

$3, S_{i}$ is restored to a default configuration with a sticky end $[\bar{s}]$, and the new color $c^{\prime}$ encoded in its duplex portion. We next describe the reactions in detail.

Step 2. In Step 2, Rule-Molecule $\tilde{R}[r]^{q c}$ hybridizes and is ligated with SymbolMolecule $[\bar{r}]^{q c} S_{i}$. The ligation product is cut into $\tilde{R}_{w}[e]^{c^{\prime}}$ (a waste molecule that diffuses away) and $[\bar{e}]^{c^{\prime}} S_{i}$. The sticky end $[\bar{e}]$ encodes the new color $c^{\prime}$. Schematically, we have,

$$
\tilde{R}[r]^{q c}+[\bar{r}]^{q c} S_{i} \rightarrow\left(R S_{i}\right)^{q c c^{\prime}} \rightarrow \tilde{R}_{w}^{q c}[e]^{c^{\prime}}+[\bar{e}]^{c^{\prime}} S_{i}
$$

Figure 4 describes the molecular implementation of Step 2 for the case when current state is $q=L O N G$, and the new color is $c^{\prime}=C_{B}$. The case for $q=S H O R T$ is similar, except that sticky end $[\bar{r}]$ of $S$ is $C \bar{x} \bar{y}$ instead of $\bar{x} \bar{y} \bar{z}$. The Rule-Molecule $\tilde{R}[r]^{q c}$ consists of three parts, in the terminology of [5], Bpm I recognition site, spacer region, and $<$ state,color $>$ detector. The $<$ state, color $>$ detector is the sticky end $[r]^{q c}$, which hybridizes with and thus detects the sticky end $[\bar{r}]^{q c}$ of the symbol molecule. The Rule-Molecule and the Symbol-Molecule are ligated and Bpm I cuts the ligation product into a waste Rule-Molecule $\tilde{R}_{w}^{q c}[e]^{c^{\prime}}$ ( $w$ for waste), which diffuses away, and a new Symbol-Molecule $[\bar{e}]^{c^{\prime}} S$, effecting the color change of the Symbol-Molecule from $c$ to $c^{\prime}$. The length of the spacer of $\tilde{R}$ (see Figure 4) determines the position of the cut in the ligation product and hence the sticky end $[\bar{e}]$ and the new color $c^{\prime}$ encoded in it. See Table 2 for the relation between the length of the spacer, the sequence of sticky end $[\bar{e}]$ and the new color $c^{\prime}$.

Step 3. The Symbol-Molecule $[\bar{e}]^{c^{\prime}} S_{i}$ obtained from Step 2 needs to be restored to its default configuration $[\bar{s}] S_{i}^{c^{\prime}}$ so that it can interact with the Head-Molecule $H_{i}$ above it when $H_{i}$ becomes active again. Note that this re-usability of the Symbol-Molecule is essential for the proper functioning of ADTM. After the restoration, the new color $c^{\prime}$ is encoded in the duplex portion of $S_{i}$, whose sticky end is the default sticky end $[\bar{s}]$ for a Symbol-Molecule: it encodes no color, but is ready to interact with an active Head-Molecule. The reaction of Step 3 is,

$$
E^{c^{\prime}}[e]^{c^{\prime}}+[\bar{e}]^{c^{\prime}} S_{i} \rightarrow(E S)^{c^{\prime}} \rightarrow E_{w}[s]+[\bar{s}] S_{i}^{c^{\prime}}
$$




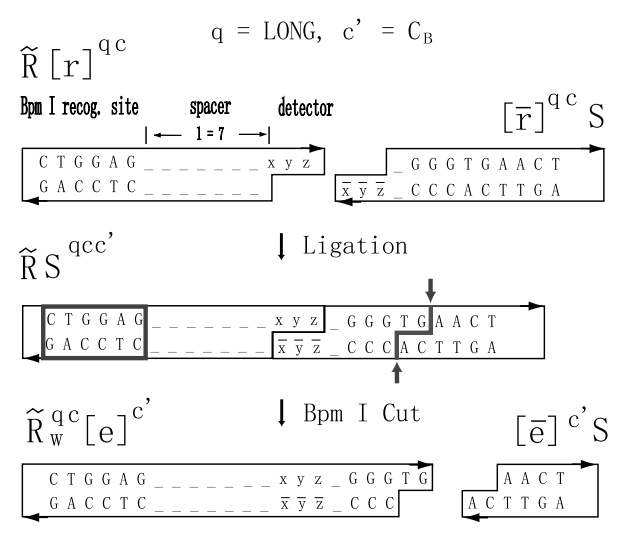

Fig. 4. Step 2 of the operation of ADTM. In this example, the current state is $q=L O N G$; the current color $q$ and state $c$ are encoded in the Symbol-Molecule's sticky end $[\bar{r}]$ whose sequence is $\bar{x} \bar{y} \bar{z}$; the new color, in this case, will be $c^{\prime}=C_{B}$, encoded in sticky end $[\bar{e}]$ whose sequence is "TG". Bpm I recognition site and cleavage site are indicated with a box and a pair of bold arrows, respectively

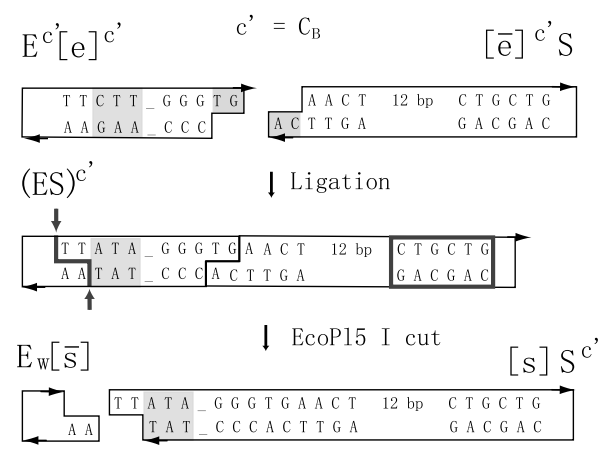

Fig. 5. Step 3 of the operation of ADTM. In this example, the new color $c^{\prime}=C_{B}$. See Figure 6 for the complete set of Assisting-Molecules $E^{c^{\prime}} e^{c^{\prime}}$. The color encoding regions are indicated with light gray background. EcoPl5 I recognition site and cleavage site are indicated with a box and a pair of bold arrows, respectively

\begin{tabular}{|c|c|c|}
\hline$c^{\prime}=C_{A}$ & $c^{\prime}=C_{B}$ & $c^{\prime}=C_{C}$ \\
\hline $\begin{array}{l}\text { T T T T A A }- \text { G G G } \\
\text { A A A A A T T C C C } \\
\end{array}$ & 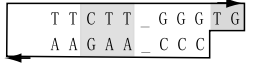 & $\begin{array}{l}\text { T T C A A }- \text { G G G T T A } \\
\text { A A G T T _ C C CA }\end{array}$ \\
\hline$c^{\prime}=C_{D}$ & $c^{\prime}=C_{E}$ & \\
\hline $\begin{array}{l}\text { T T A E E A G G G G T G } \\
\text { A A T F T T C C C C A C } \\
\end{array}$ & $\begin{array}{ll}\text { T T T C E A A } \\
\text { A A G F F T } \\
\end{array}$ & \\
\hline
\end{tabular}

Fig. 6. The complete set of Assisting-Molecules $E^{c^{\prime}}[e]^{c^{\prime}}$. The color encoding regions are indicated with light gray background 


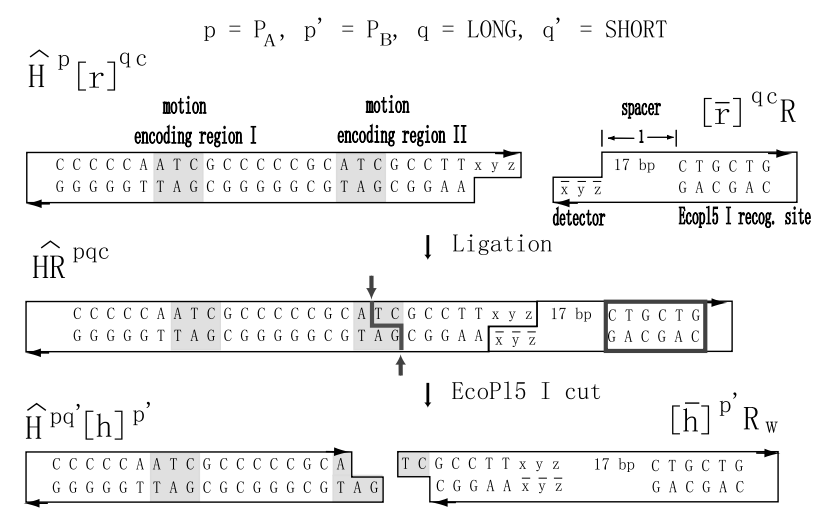

Fig. 7. Step 4 of the operation of ADTM. The motion encoding regions are indicated with light gray background. $l$ is the length of the spacer region of Rule-Molecule $R$. EcoPl5 I recognition site and cleavage site are indicated with a box and a pair of bold arrows, respectively

Figure 5 gives a molecular implementation of Step 3 for the case $c^{\prime}=C_{B}$. Color $c^{\prime}$ is encoded both in the sticky end portion and the duplex portion of Assisting-Molecule $E^{c^{\prime}}[e]^{c^{\prime}}$. Assisting-Molecule $E^{c^{\prime}}[e]^{c^{\prime}}$ detects the color encoding sticky end of SymbolMolecule $S_{i}$ and transfers its color encoding duplex portion to $S_{i}$ via ligation and subsequent cleavage. This step generates a waste product $E_{w}[s]$ that diffuses away. Note that $E_{w}[s]$ may hybridize and be ligated with some other $[\bar{s}]$ end of a Symbol-Molecules,say $[\bar{s}] S_{k}$. However, this only represents some futile reactions that will not block, reverse, or alter the operation of ADTM, since $E_{w}[s]$ will be cut subsequently away from $[\bar{s}] S_{k}$ by EcoPl 5I. Nevertheless, this does decrease the efficiency of ADTM and as the concentration of $E_{w}[s]$ increases, the negative effect on the efficiency becomes more prominent. For a complete set of Assisting-Molecules $E^{c^{\prime}}[e]^{c^{\prime}}$, see Figure 6.

Note that the existence of endonuclease EcoPl5 I recognition site in the duplex portion of $S_{i}$ adds extra complication to Step 1 and Step 2: it results in futile reactions which are discussed in [41].

\subsection{State Change of a Head-Molecule}

Step 4. In Step 4, the Head-Molecule $H_{i}^{p}[r]^{q c}$ generated in Step 2 (with its sticky end encoding the current state and color) is modified by a Rule-Molecule that decides the state transition and the motion of the head. After the modification, the new state information is encoded in the duplex portion of the modified Head-Molecule, and the motion direction of the head is encoded in the sticky end of the modified Head-Molecule in the form of a sticky end complementary to one of its neighboring Head-Molecules. The sticky end of the modified Head-Molecule will dictate it to interact with either its left or right neighbors, and thus determines the motion of the head.

More specifically, Head-Molecule $H_{i}^{p}[r]^{q c}$ hybridizes and is ligated with a free floating Rule-Molecule $[\bar{r}]^{q c} R$ and the ligation product $\left(H_{i} R\right)^{p q c}$ is cut by endonuclease EcoPl5 I into $H_{i}^{p q^{\prime}}[h]^{p^{\prime}}$ and $[\bar{h}]^{p^{\prime}} R_{w}$, a waste molecule that diffuses away. Head- 
Molecule $H_{i}^{p q^{\prime}}[h]^{p^{\prime}}$ encodes the new state $q^{\prime}$ in its duplex portion, and the motion direction $p^{\prime}$ of the head in its sticky end. The reaction of Step 4 is,

$$
\hat{H}_{i}^{p}[r]^{q c}+[\bar{r}]^{q c} R \rightarrow\left(H_{i} R\right)^{p q c} \rightarrow \hat{H}_{i}^{p q^{\prime}}[h]^{p^{\prime}}+[\bar{h}]^{p^{\prime}} R_{w}
$$

Figure 7 describes the molecular implementation for the case when the current state $q=L O N G$; new state $q^{\prime}=S H O R T$; the position type of the current Head-Molecule $H_{i}$ is $p=P_{A}$; the position type of the Head-Molecule $H_{j}$ that it will interact with is $p^{\prime}=P_{B}$ (hence $j=i+1$ in this case). The Rule-Molecule $[\bar{r}]^{q c} R$ consists of three parts: the detector sticky end $[\bar{r}]^{q c}$ that encodes the current state and color; the spacer, whose length determines the transition results (new state and motion direction of the head); and recognition site for endonuclease EcoPl5 I. The Rule-Molecule $[\bar{r}]^{q c} R$ detects the current state $q$ and color $c$ encoded in sticky end $[r]^{q c}$ of $H_{i}$ and is ligated to $H_{i}$. After ligation, endonuclease EcoP15 I cuts into the motion encoding region of the Head-Molecule and exposes a new sticky end that encodes the position type information $p^{\prime}$ ( and hence determines the motion direction). Cleavages at motion encoding regions I and II result in new states $q^{\prime}=L O N G$ and $q^{\prime}=S H O R T$, respectively. The complete set of transitions for all the combinations of different $p, q, d$, and $q^{\prime}$ is described in [41].

\subsection{Reaction between Two Adjacent Head-Molecules}

Head-Molecule $\hat{H}_{i}^{p q^{\prime}}[h]^{p^{\prime}}$ produced in Step 4 will next interact with one of its neighboring Head-Molecules, $[\bar{h}]^{p^{\prime}} H_{j}^{\prime}$, where $j=i-1$ for its left neighbor and $j=i+1$ for its right neighbor (Step 5). Then $H_{j}$ becomes an active Head-Molecule encoding the new state $q^{\prime}$ (Step 6) while $H_{i}$ is restored to its default inactive configuration (Steps 7 and 8).

Step 5. In Step 5, Head-Molecule $\hat{H}_{i}^{p q^{\prime}}[h]^{p^{\prime}}$ is ligated to either its left neighbor or its right neighbor $[\bar{h}]^{p^{\prime}} H_{j}^{\prime}$, where $j=i-1$ or $i+1$, as dictated by the $p^{\prime}$ information encoded in its sticky end. The ligation product $\left(H_{i} H_{j}\right)^{q^{\prime}}$ is cut into $H_{i}^{p}[\bar{t}]^{p p^{\prime} q^{\prime}}$ and $[t]^{p p^{\prime} q^{\prime}} \hat{H}_{j}$. The reaction of Step 5 is,

$$
\hat{H}_{i}^{p q^{\prime}}[h]^{p^{\prime}}+[\bar{h}]^{p^{\prime}} H_{j}^{\prime} \rightarrow\left(H_{i} H_{j}\right)^{q^{\prime}} \rightarrow H_{i}\left[\bar{t}^{p p^{\prime} q^{\prime}}+[t]^{p p^{\prime} q^{\prime}} \hat{H}^{\prime}{ }_{j}\right.
$$

Note that now both the sticky ends of $H_{i}$ and $H_{j}$ encode position type $p$ of $H_{i}$, position type $p^{\prime}$ of $H_{j}$, and the new state $q^{\prime}$.

Figure 8 gives a molecular implementation for this step. Panel I depicts an example case in full detail; Panel II and III show all the cases in a simplified way. Note that the sticky end $[t]$ (and $[t]$ ) encodes all the information for position type $p$ of $H_{i}$, position type $p^{\prime}$ of $H_{j}$, and the new state $q^{\prime}$, we hence have $3 \times 2 \times 2=12$ different sticky ends $[t]$.

Step 6. In Step 6, Head-Molecule $\hat{H}_{j}^{\prime}$ is modified into a Head-Molecule ready to interact with a Symbol-Molecule; in other words, it becomes an active head. The reaction of Step 6 is,

$$
\hat{H}_{j}^{\prime}[t]^{p p^{\prime} q^{\prime}}+[\bar{t}]^{p p^{\prime} q^{\prime}} T \rightarrow H_{j}^{\prime} T \rightarrow \hat{H}_{j}^{\prime q^{\prime}}[s]+[\bar{s}] T_{w}
$$


I) $\mathrm{p}=\mathrm{P}_{\mathrm{A}} ; \mathrm{p}^{\prime}=\mathrm{P}_{\mathrm{C}} ; \mathrm{q}^{\prime}=$ SHORT

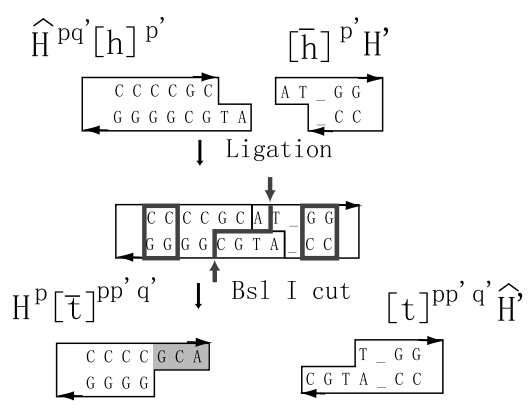

II） $\mathrm{q}^{\prime}=$ SHORT

To Right

$\mathrm{p}=\mathrm{P}_{\mathrm{A}} ; \mathrm{p}^{\prime}=\mathrm{P}_{\mathrm{B}} \downarrow$

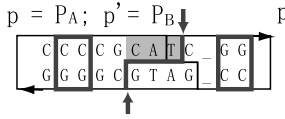

$\mathrm{p}=\mathrm{P}_{\mathrm{B}} ; \mathrm{p}^{\prime}=\mathrm{P}_{\mathrm{C} \downarrow}$

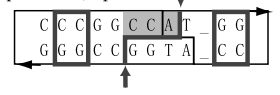

$\mathrm{p}=\mathrm{P}_{\mathrm{C}} ; \mathrm{p}^{\prime}=\mathrm{P}_{\Lambda} \downarrow$

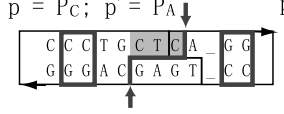

To Left

$\mathrm{p}=\mathrm{P}_{\mathrm{A}} ; \mathrm{p}^{\prime}=\mathrm{P}_{\mathrm{C}}$

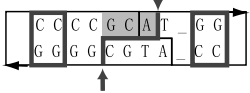

$\mathrm{p}=\mathrm{P}_{\mathrm{B}} ; \mathrm{p}^{\prime}=\mathrm{P}_{\mathrm{A}}$

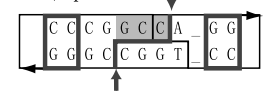

$p=P_{C} ; p^{\prime}=P_{B}$

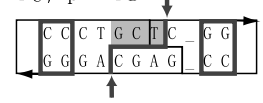

III） q' = LONG

To Right To Left

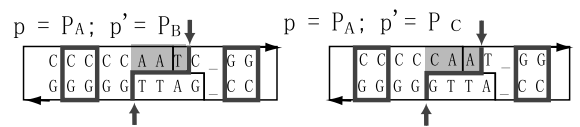

$\mathrm{p}=\mathrm{P}_{\mathrm{B}} ; \mathrm{p}^{\prime}=\mathrm{P}_{\mathrm{C} \downarrow} \quad \mathrm{p}=\mathrm{P}_{\mathrm{B}} ; \mathrm{p}^{\prime}=\mathrm{P}_{\mathrm{A}} \downarrow$

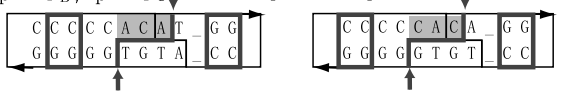

$\mathrm{p}=\mathrm{P}_{\mathrm{C}} ; \mathrm{p}^{\prime}=\mathrm{P}_{\mathrm{A}} \downarrow \quad \mathrm{p}=\mathrm{P}_{\mathrm{C}} ; \mathrm{p}^{\prime}=\mathrm{P}_{\mathrm{B}} \downarrow$

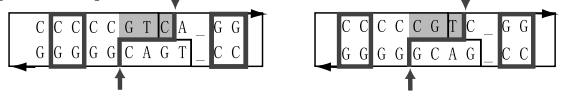

Fig. 8. Step 5 of the operation of ADTM. Panel I depicts the case when $p=P_{A}, p^{\prime}=P_{C}$, and $q^{\prime}=S H O R T$. Panel II and III describe all the cases when $q^{\prime}=S H O R T$ and all the cases when $q^{\prime}=L O N G$, respectively. In panel II and III, each case is represented in a simplified fashion that only shows the ligation product before the cleavage.Bsl I recognition sites and cleavage sites are indicated with boxes and pairs of bold arrows, respectively. The unique sticky ends $\left[\bar{t}^{p p^{\prime} q^{\prime}}\right.$ are shown with gray background

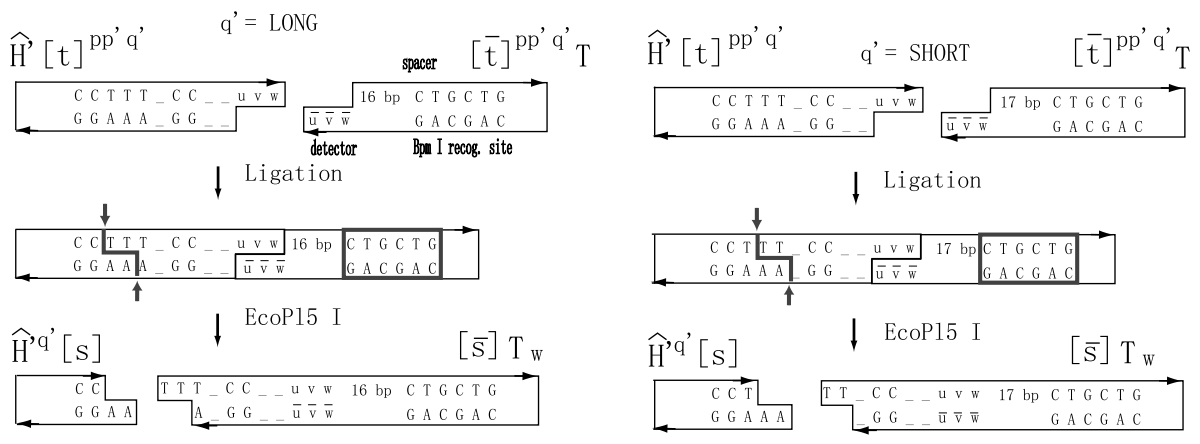

Fig. 9. Step 6 of the operation of ADTM. Bpm I recognition sites and cleavage sites are indicated with boxes and pairs of bold arrows, respectively 


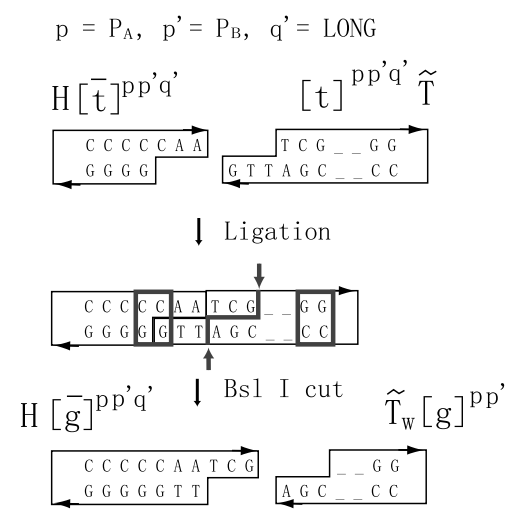

Fig. 10. Step 7 of the operation of ADTM. Bsl I recognition site and cleavage site are indicated with a box and a pair of bold arrows, respectively

Figure 9 describes the molecular implementation for Step 6. The mechanism of this step is very similar to Step 4 , and hence we omit its details.

Steps 7. and 8. In Step 7, the sticky end $\left[\bar{t}^{p p^{\prime} q^{\prime}}\right.$ of Head-Molecule $H_{i}$ is modified by an Assisting-Molecule $\tilde{T}[t]^{p p^{\prime} q^{\prime}}$ to a new sticky end $[\bar{g}]^{p p^{\prime} q^{\prime}}$. In Step 8 , the sticky end $[\bar{g}]^{p p^{\prime} q^{\prime}}$ initiates a sequential "growing-back" process which restores $H_{i}$ to its default (inactive) configuration $[\bar{h}]^{p} H_{i}^{p}$. The reaction of Step 7 is,

$$
\tilde{T}[t]^{p p^{\prime} q^{\prime}}+\left[\bar{t}^{p p^{\prime} q^{\prime}} H_{i} \rightarrow \tilde{T} H_{i} \rightarrow \tilde{T}_{w}[g]^{p p^{\prime} q^{\prime}}+[\bar{g}]^{p p^{\prime} q^{\prime}} H_{i}\right.
$$

The reaction of Step 8 is,

$$
[\bar{g}]^{p p^{\prime} q^{\prime}} H_{i} \rightarrow[\bar{h}]^{p} H_{i}^{p}
$$

Figure 10 and Figure 11 describe the molecular implementation of Step 7 and Step 8 for the case $p=P_{A}, p^{\prime}=P_{B}$, and $q^{\prime}=L O N G$, respectively. The figures are self-explanatory and hence we omit the details for brevity. Note that Step 8 is a rather spectacular process which illustrates a precisely controlled elongation mechanism using alternating ligations and cleavages. This mechanism may be of independent interest for designing other molecular devices.

\subsection{Overall Reaction Flow}

Putting all the above steps together, we have a schematic drawing for the overall flow of the reactions (Figure 12). The complete molecule set for the construction of our ADTM is described in [41].

To fully test the validity of our complex construction of ADTM, we performed computer simulation of ADTM (See http://www.cs.duke.edu/ py/paper/dnaUTM/).

\section{Technical Challenges}

Two major technical challenges in designing ADTM are to accommodate the futile reactions occurring during the operation of ADTM and to design ADTM using limited 


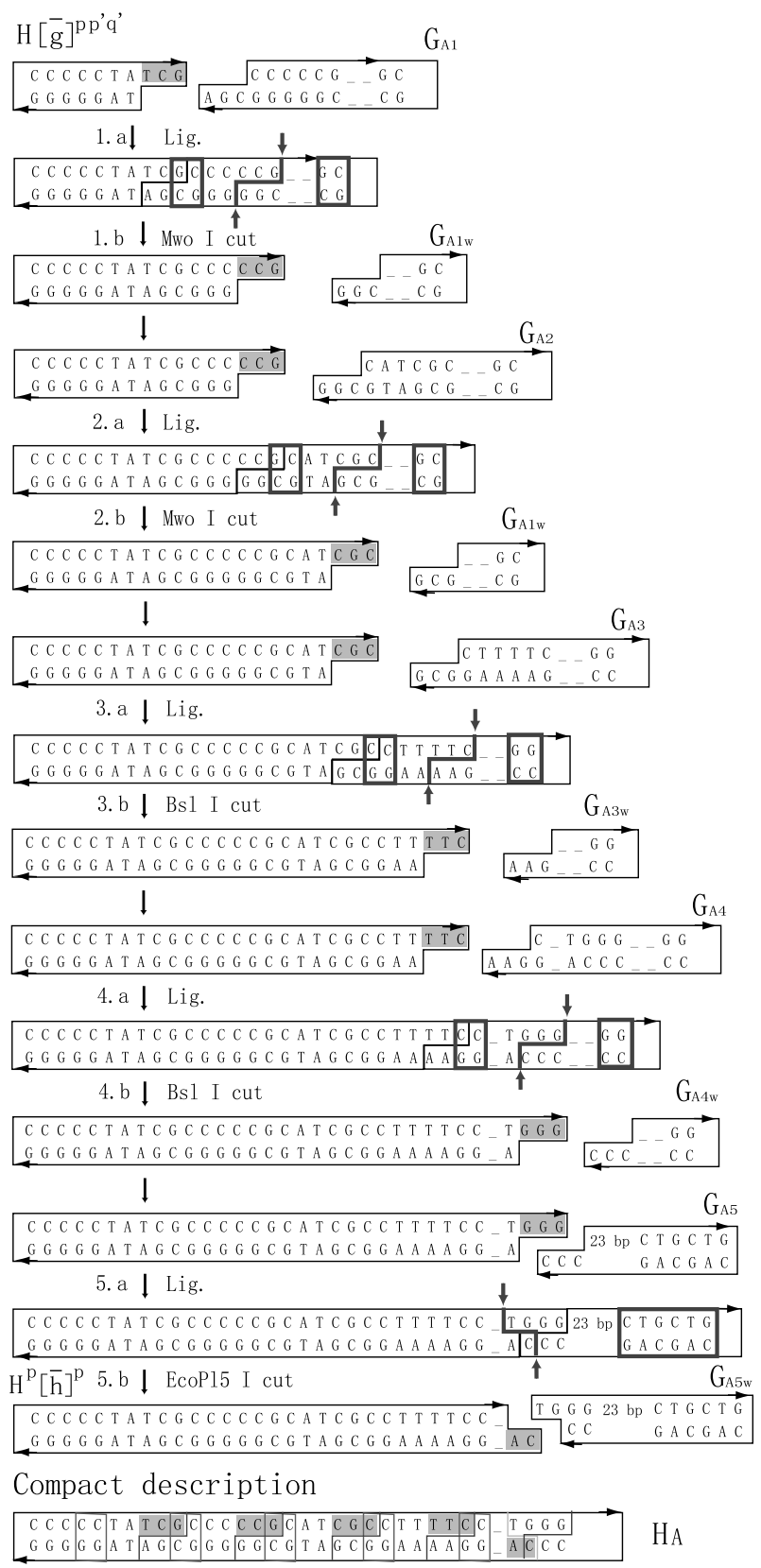

Fig. 11. Step 8 of the operation of ADTM for the case $p=P_{A}, p^{\prime}=P_{B}$, and $q^{\prime}=L O N G$. This step consists of a sequence of alternating ligations and cleavages. At each stage $k$, where $k=1-5$, the Head-Molecule is first ligated to an Assisting-Molecule $G_{A k}$ (stage $k . a$ ), then the ligation product is cut by an endonuclease (stage $k . b$ ). A waste molecule $G_{A k w}$ is generated at each stage. The last panel gives a compact representation of the whole process. The unique sticky end generated at each stage is indicated with gray background. Endonuclease recognition sites and cleavage sites are indicated with boxes and pairs of bold arrows, respectively 


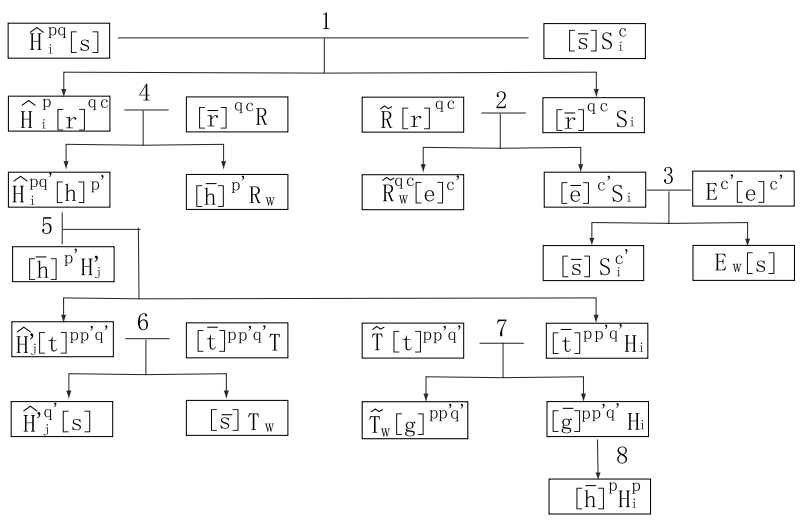

Fig. 12. Overview of the operation of ADTM

encoding space dictated by the four (six) letter vocabulary of DNA bases and by the sizes of the recognition, restriction, and spacing regions of endonucleases.

The key technique used here to address the first challenge is to make all the futile reactions fully reversible so that they do not obstruct or alter the operation of ADTM. The key technique to address the second challenge is to use an "overlay" technique as shown and to carefully select the sticky ends to avoid undesirable reactions. See [41] for details.

\section{Discussion}

In this paper, we present the design of a DNA nanomechanical device capable of universal computation and hence universal translational motion. In addition to general design principles, we give detailed molecular implementation of ADTM. A next step would be to construct a DNA cellular automata that demonstrates parallel computations.

As a consequence of the universal computation, ADTM demonstrates universal translational motion. This motion is a symbolic motion in the sense that no physical entity is moved from one location to the other. Instead, the motion is the motion of the active head symbol relative to the tracks. A nanorobotics challenge is to extend ADTM to a device that can move a physical entity, probably a DNA fragment, in a universal translational motion fashion. As a first step, it is conceivable that a DNA nanomechanical device that moves a DNA fragment bidirectionally along the track can be designed and possibly experimentally constructed.

Our complex design of ADTM makes some unconventional physical and chemical assumptions. Two lines of recent work lend partial experimental support to the practicality of this design. The first one is the autonomous DNA finite state automata constructed by Shapiro's group [3-5], in which a cascade of cleavages and ligations drive the operation of the machine. A more relevant study is the experimental construction of an autonomous unidirectional DNA walker that moves along a DNA track [42]. In this device, a walker moves unidirectionally over a sequence of three dangling anchorages 
sites (a structural analog to the dangling-molecules in ADTM design) embedded in a DNA track in an autonomous fashion, driven by alternating actions of DNA endonucleases and ligases. In particular, this walking device exploits some very similar enzyme reactions as used in the design of ADTM, such as the ligation and cleavages of DNA duplices tethered to another DNA nanostructure and the ligation of DNA fragments with 3-base overhangs at a relatively high temperature $\left(37^{\circ} \mathrm{C}\right)$.

Though a full experimental implementation of ADTM appears daunting, due to the rich set of molecules, reactions, futile reactions involved, it might be possible to experimentally test a subset of the mechanisms described here. Another challenge to experimental demonstration of ADTM is the design of an output detection mechanism.

Many futile reactions happen in the background during the operation of ADTM. A key feature of these futile reactions is that they are fully reversible. This is critical in ensuring the autonomous operation of ADTM as explained below. We initially supply the system with sufficiently high concentrations of Rule-Molecules and AssistingMolecules as well as all the byproducts generated in the futile reactions. As such, the futile reactions will reach a dynamic balance and the concentrations of all the components involved in the futile reactions, including both the "active" components essential for the operation of ADTM and the "futile" byproducts, will stay relatively constant during the operation of ADTM. Note that since the active components will not be depleted by the futile reactions (which could have happened should some futile reactions are irreversible), the autonomous operation of ADTM will not be disrupted. Though the futile reactions are innocuous, they do decrease the efficiency of ADTM. A desirable improvement of the current design is to decrease the level of futile reactions and thus increase the efficiency and robustness of ADTM.

\section{Acknowledgement}

The authors would like to thank Hao Yan and Thomas H. LaBean for helpful discussions. We are also grateful to the very helpful comments from Nabil H. Mustafa and the anonymous reviewers. This work was supported by NSF under ITR Grant EIA-0086015 and ITR Grant 0326157, by NSF under QuBIC Grant EIA-0218376 and QuBIC Grant EIA-0218359, by NSF under EMT Grant CCF-0432038 and EMT Grant CCF-0432047, by DARPA/AFSOR under Contract F30602-01-2-0561, and by RGC under Grant HKBU2107/04E.

The full version of this paper and the supplementary material can be accessed at http://www.cs.duke.edu/ py/paper/dnaUTM/.

\section{References}

1. L. Adleman. Molecular computation of solutions to combinatorial problems. Science, 266:1021-1024, 1994.

2. P. Alberti and J. L. Mergny. DNA duplex-quadruplex exchange as the basis for a nanomolecular machine. Proc. Natl. Acad. Sci. USA, 100:1569-1573, 2003.

3. Y. Benenson, R. Adar, T. Paz-Elizur, Z. Livneh, and E. Shapiro. DNA molecule provides a computing machine with both data and fuel. Proc. Natl. Acad. Sci. USA, 100:2191-2196, 2003. 
4. Y. Benenson, B. Gil, U. Ben-Dor, R. Adar, and E. Shapiro. An autonomous molecular computer for logical control of gene expression. Nature, 429:423-429, 2004.

5. Y. Benenson, T. Paz-Elizur, R. Adar, E. Keinan, Z. Livneh, and E. Shapiro. Programmable and autonomous computing machine made of biomolecules. Nature, 414:430-434, 2001.

6. Y. Chen, M. Wang, and C. Mao. An autonomous DNA nanomotor powered by a DNA enzyme. Angew. Chem. Int. Ed., 43:3554-3557, 2004.

7. D. Faulhammer, A. R. Cukras, R. J. Lipton, and L. F. Landweber. Molecular computation: RNA solutions to chess problems. Proc. Natl. Acad. Sci. USA, 97:1385 - 1389, 2000.

8. L. Feng, S. H. Park, J. H. Reif, and H. Yan. A two-state DNA lattice switched by DNA nanoactuator. Angew. Chem. Int. Ed., 42:4342-4346, 2003.

9. A. A. Henry and F. E. Romesberg. Beyond A, C, G, and T: augmenting nature's alphabet. Curr. Opin. Chem. Biol., 7:727-733, 2003.

10. T. H. LaBean, H. Yan, J. Kopatsch, F. Liu, E. Winfree, J. H. Reif, and N. C. Seeman. The construction, analysis, ligation and self-assembly of DNA triple crossover complexes. J. Am. Chem. Soc., 122:1848-1860, 2000.

11. L. F. Landweber, R. J. Lipton, and M. O. Rabin. DNA ${ }^{2}$ DNA computations: A potential 'Killer App'? In H. Rubin and D. H. Wood, editors, DNA Based Computers III: DIMACS Workshop, June 23-27, 1997, University of Pennsylvania, pages 161-172, Providence, Rhode Island, 1997. American Mathematical Society.

12. J. Li and W. Tan. A single DNA molecule nanomotor. Nano Lett., 2:315-318, 2002.

13. R. J. Lipton. DNA solution of hard computational problem. Science, 268:542-545, 1995.

14. D. Liu and S. Balasubramanian. A proton fuelled DNA nanomachine. Angew. Chem. Int. Ed., 42:5734-5736, 2003.

15. Q. Liu, L. Wang, A. G. Frutos, A. E. Condon, R. M. Corn, and L. M. Smith. DNA computing on surfaces. Nature, 403:175-179, 2000.

16. C. Mao, T. H. LaBean, J. H. Reif, and N. C. Seeman. Logical computation using algorithmic self-assembly of DNA triple-crossover molecules. Nature, 407:493-496, 2000.

17. C. Mao, W. Sun, and N. C. Seeman. Designed two-dimensional DNA holliday junction arrays visualized by atomic force microscopy. J. Am. Chem. Soc., 121:5437-5443, 1999.

18. C. Mao, W. Sun, Z. Shen, and N. C. Seeman. A DNA nanomechanical device based on the B-Z transition. Nature, 397:144-146, 1999.

19. Q. Ouyang, P. D. Kaplan, S. Liu, and A. Libchaber. DNA solution of the maximal clique problem. Science, 278:446-449, 1997.

20. J. H. Reif. Parallel molecular computation: Models and simulations. In Proceedings: 7 th Annual ACM Symposium on Parallel Algorithms and Architectures (SPAA'95) Santa Barbara, CA, pages 213-223, 1995.

21. J. H. Reif. Paradigms for biomolecular computation. In C. S. Calude, J. Casti, and M. J. Dinneen, editors, First International Conference on Unconventional Models of Computation, Auckland, New Zealand, pages 72-93. Springer Verlag, 1998.

22. J. H. Reif. Local parallel biomolecular computation. In H. Rubin and D. H. Wood, editors, DNA-Based Computers 3, volume 48 of DIMACS, pages 217-254. American Mathematical Society, 1999.

23. J. H. Reif. The design of autonomous DNA nanomechanical devices: Walking and rolling DNA. Lecture Notes in Computer Science, 2568:22-37, 2003. Published in Natural Computing, DNA8 special issue, Vol. 2, p 439-461, (2003).

24. P. W. K. Rothemund. A DNA and restriction enzyme implementation of Turing machines. In R. J. Lipton and E. B. Baum, editors, DNA Based Computers: Proceedings of the DIMACS Workshop, April 4, 1995, Princeton University, volume 27, pages 75 - 119, Providence, Rhode Island, 1996. American Mathematical Society.

25. A. J. Ruben and L. F. Landweber. The past, present and future of molecular computing. Nature Rev. Mol. Cell Biol., 1:69-72, 2000. 
26. N. C. Seeman. DNA in a material world. Nature, 421:427-431, 2003.

27. W. B. Sherman and N. C. Seeman. A precisely controlled DNA biped walking device. Nano Lett., 4:1203-1207, 2004.

28. F. C. Simmel and B. Yurke. Using DNA to construct and power a nanoactuator. Phys. Rev. E, 63:041913, 2001.

29. F. C. Simmel and B. Yurke. A DNA-based molecular device switchable between three distinct mechanical states. Appl. Phys. Lett., 80:883-885, 2002.

30. W. D. Smith. DNA computers in vitro and in vivo. In R. J. Lipton and E. B. Baum, editors, DNA Based Computers: Proceedings of the DIMACS Workshop, April 4, 1995, Princeton University, pages 121 - 186, Providence, Rhode Island, 1996. American Mathematical Society.

31. A. J. Turberfield, J. C. Mitchell, B. Yurke, Jr. A. P. Mills, M. I. Blakey, and F. C. Simmel. DNA fuel for free-running nanomachines. Phys. Rev. Lett., 90:118102, 2003.

32. A. M. Turing. On computable numbers, with an application to the Entscheidungs problem. In Proc. London Math. Society Ser. II, volume 42 of 2, pages 230-265, 1936.

33. A. M. Turing. On computable numbers, with an application to the entscheidungsproblem. In Proc. London Math. Society Ser. II, volume 43, pages 544-546, 1937.

34. E. Winfree. On the computational power of DNA annealing and ligation. In R. J. Lipton and E. B. Baum, editors, DNA Based Computers 1, volume 27 of DIMACS, pages 199-221. American Mathematical Society, 1996.

35. E. Winfree, F. Liu, L. A. Wenzler, and N. C. Seeman. Design and self-assembly of twodimensional DNA crystals. Nature, 394:539-544, 1998.

36. S. Wolfram. A new kind of science. Wolfram Media, Inc., Champaign, IL, 2002.

37. H. Yan, T. H. LaBean, L. Feng, and J. H. Reif. Directed nucleation assembly of DNA tile complexes for barcode patterned DNA lattices. Proc. Natl. Acad. Sci. USA, 100:8103-8108, 2003.

38. H. Yan, S. H. Park, G. Finkelstein, J. H. Reif, and T. H. LaBean. DNA-templated selfassembly of protein arrays and highly conductive nanowires. Science, 301:1882-1884, 2003.

39. H. Yan, X. Zhang, Z. Shen, and N. C. Seeman. A robust DNA mechanical device controlled by hybridization topology. Nature, 415:62-65, 2002.

40. P. Yin, A. J. Turberfield, and J. H. Reif. Designs of autonomous unidirectional walking DNA devices. In DNA Based Computers 10, 2004.

41. P. Yin, A. J. Turberfield, S. Sahu, and J. H. Reif. Design of an autonomous DNA nanomechanical device capable of universal computation and universal translational motion. Technical Report CS-2004-07, Duke University, Computer Science Department, 2004.

42. P. Yin, H. Yan, X. G. Daniell, A. J. Turberfield, and J. H. Reif. A unidirectional DNA walker moving autonomously along a linear track. Angew. Chem. Int. Ed., 2004. In press.

43. B. Yurke, A. J. Turberfield, Jr. A. P. Mills, F. C. Simmel, and J. L. Neumann. A DNA-fuelled molecular machine made of DNA. Nature, 406:605-608, 2000. 